\title{
Statistical variability of top of atmosphere cloud-free shortwave aerosol radiative effect
}

\author{
T. A. Jones and S. A. Christopher \\ Department of Atmospheric Sciences, The University of Alabama in Huntsville, Huntsville, AL, USA \\ Received: 11 December 2006 - Published in Atmos. Chem. Phys. Discuss.: 8 March 2007 \\ Revised: 25 May 2007 - Accepted: 27 May 2007 - Published: 12 June 2007
}

\begin{abstract}
The statistical variability of globally averaged MODIS aerosol optical thickness at $0.55 \mu \mathrm{m}$ (AOT) and top of atmosphere CERES cloud-free shortwave radiative effect (SWRE) is presented. Statistical variability is defined as the robustness of globally averaged statistics relative to data distribution. At the CERES footprint level, which we label "raw data", both the AOT and SWRE data derived from clear-sky CERES-SSF products show significant deviations from a normal distribution as evidenced by high skewness values. The spatial and temporal distribution of the data is also not uniform, with a greater concentration of data occurring in aerosol heavy-regions. As a result, globally averaged AOT and SWRE are overestimated when derived from raw data alone. To compensate, raw data are gridded into $2 \times 2$ degree grid-cells (called "gridded" data) to reduce the effect of spatial non-uniformity. However, the underlying non-normal distribution remains and manifests itself by increasing the uncertainty of grid-cell values. Globally averaged AOT and SWRE derived from a gridded dataset are substantially lower than those derived from raw data alone. The range of globally averaged AOT and SWRE values suggests that up to a $50 \%$ statistical variability exists, much of which is directly tied to how the data are manipulated prior to averaging. This variability increases when analyzing aerosol components (e.g. anthropogenic) since component AOT (and SWRE) may not exist at all locations were AOT is present. As a result, regions where a particular component AOT does not exist must either not be included in the global average or have data within these regions set to null values. However, each method produces significantly different results. The results of this work indicate simple mean and standard deviation statistics do not adequately describe global aerosol climate forcing data sets like the one used here. We demonstrate that placing raw observations on to a uniform grid is a
\end{abstract}

Correspondence to: S. A. Christopher

(sundar@nsstc.uah.edu) necessary step before calculating global statistics. However, this by no means eliminates uncertainty in globally averaged AOT and SWRE values, while adding its own set of assumptions. When reporting any globally averaged statistic, it is important to report corresponding distribution and coverage information, in the form of skewness values, probability density functions, and spatial distribution plots, to help quantify its usefulness and robustness.

\section{Introduction}

The difference between top of atmosphere (TOA) clear and aerosol skies in the shortwave $(<4 \mu \mathrm{m})$ portion of the electromagnetic spectrum for all aerosols is called the shortwave aerosol radiative effect (SWRE). Previous studies have used various methods to determine the global, ocean-only, TOA SWRE (see Anderson et al., 2005, and Yu et al., 2006, for reviews). These studies report a globally (and diurnally) averaged SWRE between -4.6 and $-5.4 \mathrm{~W} \mathrm{~m}^{-2}$. The negative value indicates that over global oceans the aerosols are more reflective than the background in contrast to the greenhouse gas forcing values that are positive. The uncertainties associated with this number include instrument calibration, quality of angular models for converting shortwave (SW) radiance into flux, the effect of cloud contamination, assumptions in aerosol and surface properties (Zhang and Reid, 2006), and the assumptions made during the calculation of a clearsky, aerosol free SW flux background, have been well documented (Zhang et al., 2005a). These uncertainties can account for a variation in SWRE of approximately $30 \%$ in some instances (Anderson et al., 2005; Zhang et al., 2005a).

However, one source of uncertainty (or variability) in globally averaged values has received much less attention, namely the implicit assumptions made when reporting a globally averaged statistic. Both TOA SW flux and aerosol optical thickness (AOT) vary substantially over the large

Published by Copernicus GmbH on behalf of the European Geosciences Union. 
spatial and temporal domains used by these studies. An "average" value is only representative if the source data has a normal (or Gaussian) distribution (Wilks, 2006). In this paper we will show that SWRE is not normally distributed, and will note the importance of evaluating additional statistical measures in describing a global data set.

Additional statistical variability originates from the manner in which the data are manipulated prior to reporting the global average. Satellite derived datasets such as that used by Christopher and Zhang (2002) do not have a uniform spatial distribution due to satellite orbit and sensor observing characteristics. They used CERES pixel level data that has a nominal spatial resolution of $20 \mathrm{~km}$ at nadir that also contains the MODIS AOT convolved within the CERES pixel. The term "raw data" refers to AOT and SWRE values derived from individual CERES-resolution pixels. To reduce the effect of the non-uniform spatial distribution, raw data are gridded (generally to a $2 \times 2$ latitude-longitude grids) that we call "gridded data" using the assumption that each grid-cell caries an equal weight during the calculation process. While the use of gridded data can reduce the effect of a non-uniform spatial distribution of these statistics (assuming some data exist for all grid-cells), each grid-cell within the gridded data set has a different statistical meaning. Since the raw data are not uniformly distributed, each grid-cell contains differing numbers of raw pixels. Over a global domain, the number of pixels used in each grid cell can vary from a single pixel to over a thousand. The effect of outliers present within sparse data regions will be magnified, increasing the uncertainty of the SWRE reported in these regions.

Another common statistic used to describe global radiative effect is the "aerosol radiative efficiency $\left(E_{\tau}\right)$ ". $E_{\tau}$ is defined simply as the SWRE divided by AOT. This value can either be calculated on a pixel-by-pixel (or grid-cell) basis or simply calculated using one global mean SWRE and AOT value. Since this statistic is calculated by dividing a very small number (AOT) into a number two orders of magnitude greater (SWRE), $E_{\tau}$ is very sensitive to small variations in AOT. For example, Christopher and Zhang (2002) reported an average maritime AOT of 0.04 with a corresponding SWRE of $-0.9 \mathrm{~W} \mathrm{~m}^{-2}$, resulting in an efficiency of $-22 \mathrm{~W} \mathrm{~m}^{-2} \tau^{-1}$. Other research (Kaufman et al., 2005a) note a maritime AOT of 0.06 , which if used to calculate $E_{\tau}$ would result in a value of $-15 \mathrm{~W} \mathrm{~m}^{-2} \tau^{-1}$. Thus, even a small error in AOT can have a large impact on reported $E_{\tau} . E_{\tau}$ can also be defined as the linear regression coefficient between SWRE and AOT. This value is much more resilient to changes in AOT average and provides a method to determine the uncertainty of this statistic.

A further complication arises when analyzing SWRE as a function of aerosol type. Using the technique developed by Kaufman et al. (2005a, b), total aerosol optical thickness can be separated into maritime, dust, and anthropogenic components. However, this algorithm can produce negative dust or anthropogenic AOT, which has no physical meaning.
Thus, the assumption is made that an aerosol component is not present when its component AOT is less than or equal to zero. This is a perfectly reasonable assumption as some areas are dominated by certain aerosol types (e.g. dust in the eastern Atlantic). The complication arises when computing global averages of component AOT and SWRE and determining regions where a particular aerosol component exists. Two possibilities are examined. First, pixels where component AOT does not exist will be ignored and global averages computed. Another method used by Christopher et al. (2007), set anthropogenic AOT and SWRE equal to zero where anthropogenic aerosols are not present, though it was not explicitly stated. The implications of this assumption will be explored in Sects. 3 and 4.

Various averaging techniques, which are described in Sect. 3, will be compared to document the statistical variability in SWRE as a function of method used. Advantages and disadvantages of each technique will be analyzed to determine which best represents global SWRE. All SWRE values reported throughout this work will be instantaneous. No diurnal or sample bias adjustments (Sample bias exists when using coarse spatial resolution data sets when compared to higher resolution MODIS data) will be applied (Zhang et al., 2005b). This is done to maintain focus on the statistical properties of the dataset without introducing the additional uncertainties associated with the various adjustment techniques.

\section{Data}

\subsection{Terra CERES SSF data}

We utilize 12 months of the CERES Single Satellite Footprint (SSF) FM1, Edition 2B data from the Terra polar orbiting satellite (December 2000-November 2001) over the global oceans between $\pm 60^{\circ}$ latitude. The CERES SSF product contains the point spread function weighted MODIS (Collection 4) aerosol and cloud properties for each CERES footprint. The CERES SSF reports measured TOA radiances, which are inverted to fluxes using angular dependence models (ADM's). We use the Terra ADM's derived by Zhang et al. (2005b), which are a function of AOT, surface wind speed and fine mode fraction. Use of ERBE or TRMM ADM's in place of Terra ADMs can affect global instantaneous SWRE by $\pm 1 \mathrm{~W} \mathrm{~m}^{-2}$ (Zhang et al., 2005a).

The SWRE is calculated by subtracting CERES SW flux where aerosols are present from clear-sky flux. "Clear-sky" is the definition used for regions that are cloud and aerosolfree. Cloud-free CERES level pixels are defined as those with $>99 \%$ CERES clear-sky fraction and with the separately measured MODIS cloud fraction $\leq 1 \%$ (for viewing and solar zenith angles $<60^{\circ}$ ). Since perfectly aerosol-free conditions never exist, clear-sky flux values are calculated on a CERES pixel-by-pixel basis by assuming that a linear relationship exists between cloud-free AOT and TOA flux for 
$\tau_{0.55}<0.4$. For $6^{\circ}$ solar zenith angle bins, the regression coefficient and constant between AOT and SW flux are computed. $\mathrm{F}_{\mathrm{clr}}$ flux is derived by subtracting the AOT*slope values from the CERES fluxes (Zhang et al., 2005a).

\subsection{Dust, maritime, and anthropogenic AOT components}

To determine the maritime, dust, and anthropogenic components of the total TOA SWRE, the CERES footprint AOT $\left(\tau_{0.55}\right)$ is separated into three components including maritime sea spray $\left(\tau_{m a}\right)$, atmospheric dust $\left(\tau_{d u}\right)$, and anthropogenic aerosols $\left(\tau_{a n}\right)$ (Eq. 1) based on Kaufman et al. (2005a).

$\tau_{0.55}=\tau_{m a}+\tau_{a n}+\tau_{d u}$

The maritime contribution can be estimated using surface wind speed $(\mathrm{W})$ derived from the European Centre for Medium-Range Weather Forecasts (ECMWF) that is reported within the CERES SSF product (Eq. 2).

$\tau_{m a}=0.007 \mathrm{~W}\left(\mathrm{~ms}^{-1}\right)+0.02$

The MODIS total fine mode fraction $(f)$, can be separated into the same three components where $f_{m a}, f_{d u}$, and $f_{a n}$ correspond to the fine mode fraction of maritime, dust, anthropogenic aerosols respectively (Eq. 3).

$f=\left[f_{m a} \tau_{m a}+f_{d u} \tau_{d u}+f_{a n} \tau_{a n}\right] / \tau_{0.55}$

Dust AOT can then be derived by combining Eqs. (1-3) and solving for $\tau_{d u}$ (Eq. 4) using the assumption that $f$ is bounded by: $\left(f_{m a} \leq f \leq f_{a n}\right)$. Anthropogenic AOT are calculated by subtracting $\tau_{d u}$ and $\tau_{m a}$ from $\tau_{0.55}$, which is consistent with Christopher et al. (2006).

$\tau_{d u}=\left[\tau_{0.55}\left(f_{a n}-f\right)-\tau_{m a}\left(f_{a n}-f_{m a}\right)\right] /\left(f_{a n}-f_{d u}\right)$

To calculate $\tau_{d u}$, values for $f_{m a}, f_{d u}$, and $f_{a n}$ are required. Kaufman et al. (2005) estimated these values for the tropical southern Atlantic $\left(0-30^{\circ} \mathrm{S}\right)$ and reported an error $10-15 \%$ in the retrieved dust AOT (Eq. 5).

$$
f_{m a}=0.3 \pm 0.1, f_{d u}=0.5 \pm 0.05, f_{a n}=0.9 \pm 0.05
$$

SWRE is then scaled by the ratio of component AOT to total AOT to determine the SWRE for each aerosol component (Christopher and Jones, 2007).

\section{Averaging methods}

\subsection{Simple average}

The first averaging method to be analyzed is the sample mean, which is also the simplest and most readily used statistic to describe large datasets (Wilks, 2006). The mean, or average, is defined as simply the sum of the sample data values $\left(x_{i}\right)$ divided by the total sample size (n) (Eq. 6). The resulting average is generally thought of as a central value, which best describes a total dataset. Information about the variability of the data around the mean value can be determined by calculating its standard deviation (Eq. 7). Often, error bars are defined as ranging one or two standard deviations from the sample mean.

$\bar{x}=\frac{1}{n} \sum_{i=1}^{n} x_{i}$

$s=\sqrt{\frac{1}{n-1} \sum_{i=1}^{n}\left(x_{i}-\bar{x}\right)^{2}}$

Unfortunately, the mean statistic is only adequate when the source data is normally distributed, which is rather uncommon for atmospheric datasets (Wilks, 2006). To compensate, some have chosen to use the median statistics, which is more robust for non-normally distributed datasets. However, it still does not provide any information concerning distribution, therefore the median is not a focus of this study. The skewness statistic can be used to determine the degree to which a dataset does not conform to a normal distribution (Eq. 8).

$\gamma=\frac{\frac{1}{n-1} \sum_{i=1}^{n}\left(x_{i}-\bar{x}\right)^{3}}{s^{3}}$

A positive value of the skewness statistic indicates that the data is biased towards low values with fewer larger values, which manifest themselves on the right-hand side of a probability density function. Conversely, negative values of skewness indicate the presence of many large value data points, with fewer small values. Skewness values larger than $\pm 2 *$ Standard error of skewness (SEK) indicate that a particular data distribution cannot be considered normal, that also indicates that basic mean and standard deviation statistics may not be reliable. The standard error of skewness can be estimated by Eq. (9).

$\mathrm{SEK}=\sqrt{\frac{6}{n}}$

The CERES-SSF AOT and SWRE datasets used here are also not homogeneously distributed over space and time that can potentially bias the results toward a space and time where observed data may be more common. None of these statistics take into account the natural spatial and temporal variability present in a global dataset.

The radiative efficiency $\left(E_{\tau}\right)$ will also be calculated from the raw data set. The SWRE/AOT and linear regression methods for calculating $E_{\tau}$ will be compared. One advantage of this statistic is that the SWRE-AOT relationship should remain fairly insensitive to the temporal and spatial variations within the data (for a constant solar zenith angle and similar aerosol characteristics). A regional analysis by Jones and Christopher (2007b) showed that $E_{\tau}$ varied less than $\pm 5 \mathrm{~W} \mathrm{~m}^{-2} \tau^{-1}$ for 9 out of 10 study regions. 


\subsection{Random sampling}

To determine the resiliency to outliers of the simple average technique, a subset of pixels is randomly sampled and statistics from that random sample computed. For this analysis, each random sample consists of 5\% of the total number of raw pixels. Mean AOT and SWRE are computed from each random sample. This process is repeated 500 times to determine the stability of the overall average. Smaller variations would indicate that the overall data is resilient to outliers, which would appear in some, but not all the random samples. The resulting statistics could then be used to define a "sampling uncertainty", one of many uncertainties actually present. However, even if this sample uncertainty proves low, it may not adequately describe the data due to the distribution issues discussed above. Also, the random samples used here will still have the same spatial and temporal variations present in the complete dataset.

\subsection{Gridded data}

To reduce the effect of large spatial inhomogeneities in the raw data, the data are binned into a uniform grid of $2 \times 2$ degree latitude-longitude cells. Each grid-cell value represents the simple average of all raw pixels located within that particular cell. The standard deviation, skewness, and number of raw pixels within each grid-cell are also recorded. There are several advantages to gridding. The biggest advantage is that it produces a quasi-spatially homogeneous dataset from data that was previously non-uniformly distributed in space. We define "spatially homogenous" data as data that has an equal number of data points for equal spatial domains. However, we recognize that the number of pixels used within each grid-cell remains quite inhomogeneous. Using gridded data, additional parameters such as the areal coverage of a certain aerosol type can be calculated. Global AOT and SWRE statistics are recomputed from the gridded data set are compared with those computed from the raw data set.

However, gridding data is not a cure-all to the distribution issues present in the raw data. Recall that each grid-cell is calculated as the mean of all pixels that fall within its boundaries. However, the number of raw pixels that may lie within a particular grid-cell may vary substantially, from only a few to over 1000 . In sparse data regions, grid-cell values are defined by only a few pixels. As a result, outliers in these regions can significantly affect the grid cell value, causing it to now be a grid-cell outlier. Using a threshold for the number of pixels required in a grid-cell was considered, but not applied because even a small threshold number $(\sim 50)$ would eliminate data for several large regions. Since the gridded data sample size is much smaller than the raw sample size, the effect of missing data would be magnified in the globally averaged statistics. Another problem occurs when 1000 or more data points may be available for a particular grid cell. Here, a few outliers are not the problem, but the distribution of the data within that grid-cell is. Like the raw data, the data used in a particular grid cell may not be normally distributed. Recall that a grid-cell value is the simple mean of all raw data within its boundaries, and that a mean statistic is only robust when the data is normally distributed. If a normal distribution does not exist, then the resulting grid cell values will be biased one way or the other. This bias will in turn influence any statistics derived from a gridded dataset.

\subsection{Random gridding}

As with the raw data, the gridded data are tested using a random sample analysis to determine its stability and resilience to outliers. Grid cell values are computed by randomly selecting three raw data pixels within a $2 \times 2$ degree bin and taking the average. (Larger random samples were not used since they would have required the removal of a significant number of grid-cells from this analysis). This is repeated for each grid cell in the global field and global statistics computed. The entire process is repeated 100 times to determine the sample uncertainty of the gridded data values. It must be noted that since the raw data is not uniformly distributed, some grid cells will be more "random" than others. The three random pixels used for each grid cell mean always originate from the original number of pixels present within that grid cell, whether it is 10 or 1000 . Thus, grid cells that encompass a larger number of raw data points will have a larger variation in values owing to a greater number of random combination of points possible within these grid-cells. As a result, only a subset of grid-cell values will vary significantly with each random perturbation. Still, this analysis will show if the variations in those grid-cells represent a significant effect to the overall gridded statistics.

\subsection{What if anthropogenic AOT do not exist?}

An additional complication arises when components of AOT SWRE are analyzed. Since, some components (e.g. dust or anthropogenic) may not exist for all pixels and grid-cells, each component sample size will differ. One solution, used by Christopher et al. (2006) for anthropogenic aerosols, is to set component AOT and SWRE equal to zero when that component was not present, but a total radiative effect value does exist.

However, this method presents several problems. The first is that the assumption of AOT and radiative effect equal to zero where the Kaufman et al. (2005a, b) technique (Eq. 4) fails may lead to an under-estimation of component values. For example, if anthropogenic aerosols only exist for $70 \%$ of data where any aerosol type exist, then the remaining $30 \%$ in the anthropogenic average will be comprised of zeros (or null data), substantially lowering the average. The argument can be made that if an aerosol component does not exist, then its values should be set to zero. However, the matter really depends on what one is trying to show. For example, if one 

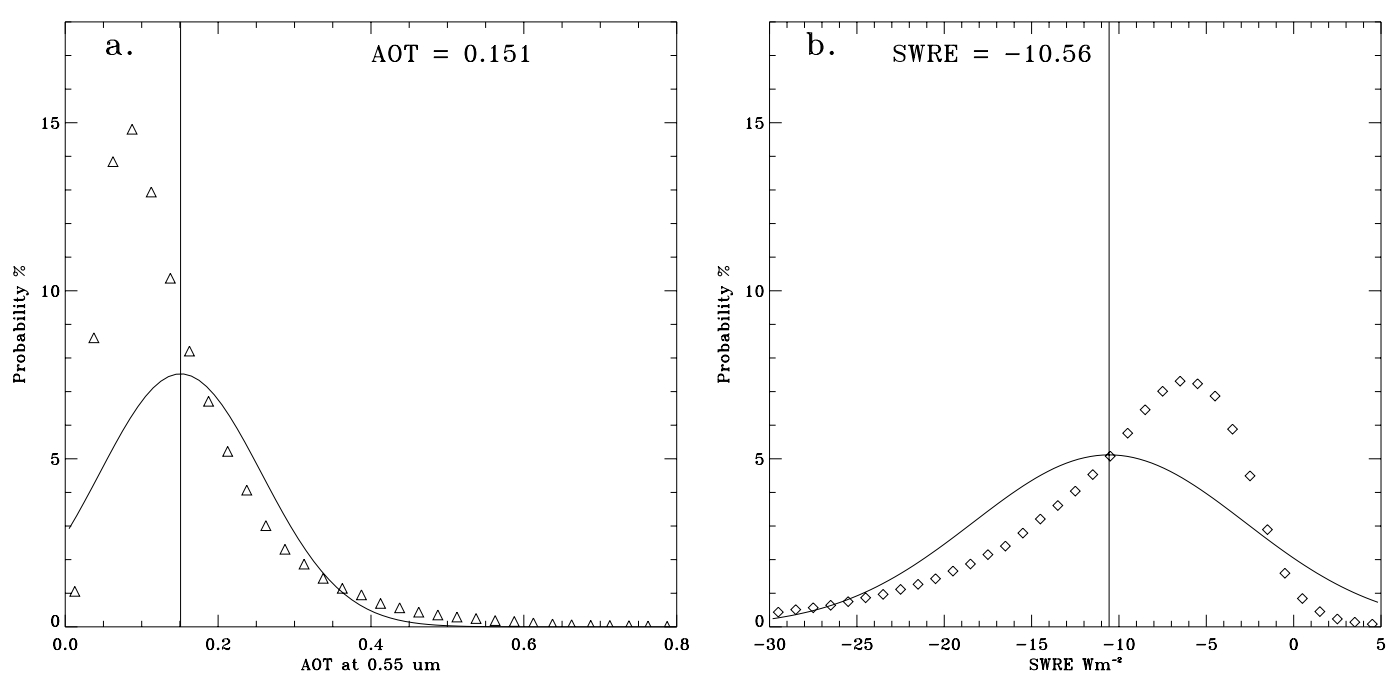

Fig. 1. Probability density functions of non-gridded, global AOT (a) and SWRE (b) shown by symbols with idealized Gaussian (normal) distributions overplotted (solid line). Bin sizes are 0.01 and $0.2 \mathrm{~W} \mathrm{~m}^{-1}$ for AOT and SWRE respectively. Vertical lines indicate mean values for AOT and SWRE, respectively.

is only interested in analyzing dust SWRE independently of other aerosols, it makes sense to only analyze data where dust aerosols are actually present (e.g. Christopher and Jones, 2007). Conversely, if one is trying to determine the relative importance of maritime, dust, and anthropogenic aerosols to SW radiative effect, then the creation of homogenous component datasets using the method described above becomes necessary.

The other significant problem introduced by setting component values equal to zero occurs when these data are gridded. Null data can contribute a large proportion of data to some grid-cells, increasing its influence and further removing the data from a normal distribution. This is especially true in sparse data regions where only a few data points feed into grid-cell values. When including null component data, AOT and SWRE values for these grid cells will be decreased substantially compared to pixels with a larger concentration of data that included all AOT components. The net result is that average component values derived from gridded data will be significantly lower than there non-gridded counterparts. This underestimation of component SWRE may bias a researcher's conclusions as to the importance of a particular AOT component. The effect of the null data assumption of component AOT and radiative effect statistics will be explored for both raw and gridded datasets.

\section{Results}

\subsection{Simple average}

Using non-gridded data, the globally averaged MODIS AOT in the CERES-SSF and instantaneous SWRE are 0.15 and $-10.6 \mathrm{~W} \mathrm{~m}^{-2}$, respectively (Table 1 ). The large spatial and
Table 1. Gridded and non-gridded global AOT and SWRE raw data count, with corresponding mean, standard deviation, and skewness statistics.

\begin{tabular}{lrrrr}
\hline No-Grid & $N$ & Mean & Stddev & Skewness \\
\hline AOT & 968030 & 0.151 & 0.106 & 1.8 \\
SWRE & 968030 & -10.56 & 7.8 & -1.47 \\
Grid & & & & \\
AOT & 7412 & 0.117 & 0.061 & 2.4 \\
SWRE & 7412 & -8.01 & 4.145 & -2.17 \\
\hline
\end{tabular}

temporal variations within these data lead to large sample standard deviations of 0.11 and 7.8, exceeding all known uncertainties present within the data, though given the spatial and temporal variability of these parameters, large standard deviation values were expected. The skewness statistics for AOT and SWRE indicate that the data are not normally distributed, with values greatly exceeding $2 \times$ SEK $(0.01)$ (Table 1). The mean values for both AOT and SWRE are displaced from the peak of the PDF functions in Fig. 1, also indicative of a non-normal distribution. The AOT data trend towards smaller values, resulting in something approximating a Gamma distribution with a small shape parameter (Fig. 1a). The SWRE is biased in the opposite direction with a greater probability of pixels values occurring where the radiative effect is weak (e.g. near zero) (Fig. 1b). Interestingly, SWRE has a somewhat smaller skewness factor (in an absolute sense) than does AOT (Table 1). As a result, the globally averaged instantaneous SWRE value derived from raw data $\left(-10.6 \mathrm{~W} \mathrm{~m}^{-2}\right)$ is substantially larger than those $(-6.4$, $-7.6 \mathrm{~W} \mathrm{~m}^{-2}$ ) reported by previous studies (e.g. Christopher 

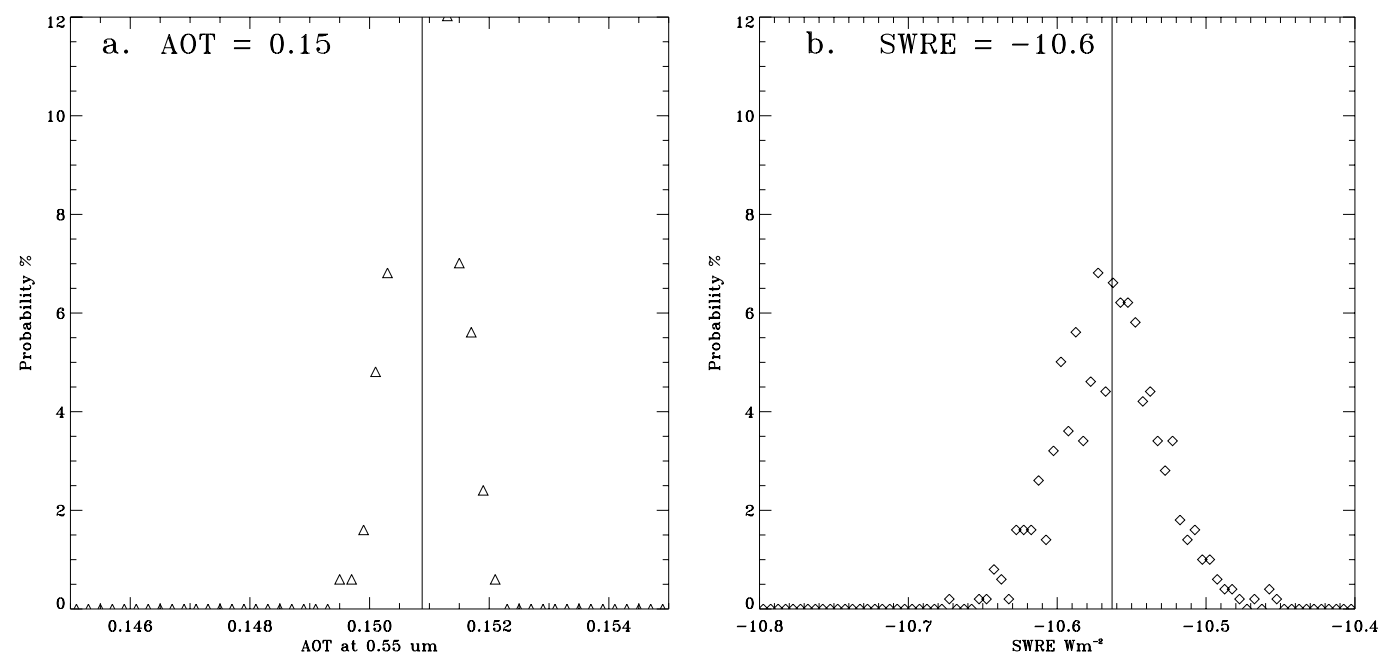

Fig. 2. Probability density functions of mean AOT (a) and SWRE (b) derived by randomly sampling $5 \%$ of the total number of pixels and computing the average, and repeating the process 500 times. Vertical lines indicate mean values for AOT and SWRE, respectively.

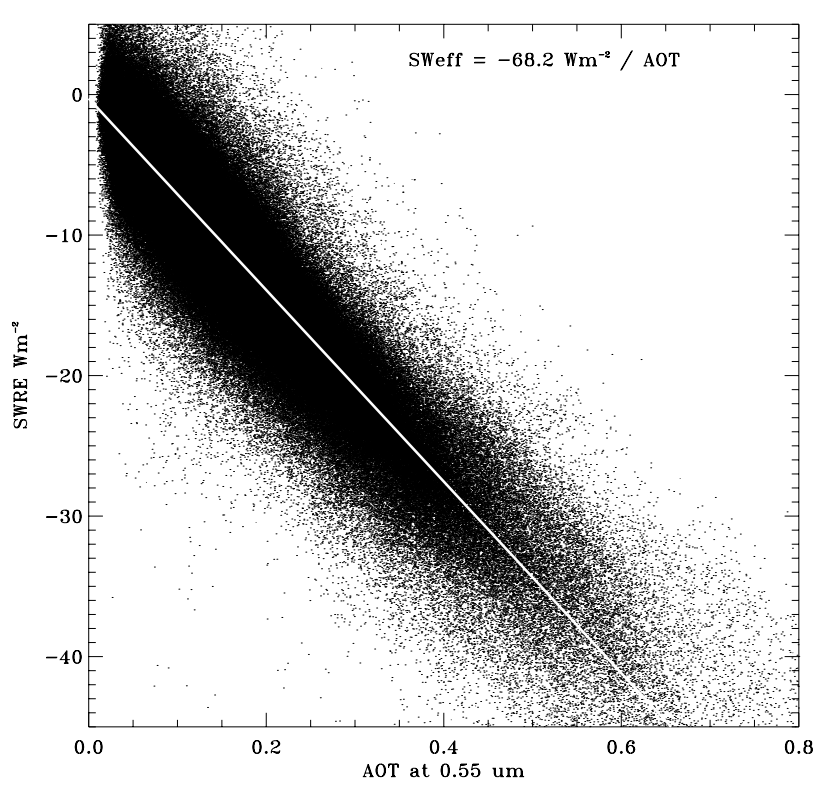

Fig. 3. Scatterplot of AOT vs. SWRE with linear regression line overplotted.

and Zhang, 2002; Loeb and Manalo-Smith, 2005). Note that the diurnal adjustment has been removed from the Loeb and Manalo-Smith (2005) value for comparison to the instantaneous values use here. The values associated with the peaks in the AOT and SWRE PDF functions are approximately 0.10 and $-7.0 \mathrm{~W} \mathrm{~m}^{-2}$, respectively, much closer to accepted values.

A comparison of average AOT and SWRE, calculated from 500 random simulations, shows that the overall statis- tic is not sensitive to outliers. The variation in AOT is only $0.11 \pm 0.0005$ and is normally distributed (Fig. 2a). Similarly, the variation in SWRE is also very small, \pm 0.034 (Fig. 2b). Since, these variations are well within $1 \%$ of the mean value, the sample data can be declared robust against outliers. Even though the sample average is stable, each random sample has the same distribution and non-uniformity issues present in the total dataset. Thus, these values remain an overestimate of global AOT and SWRE.

Using the standard definition of $E_{\tau}$ (SWRE $\div$ AOT), the instantaneous global $E_{\tau}$ is $-70.7 \mathrm{~W} \mathrm{~m}^{-2} \tau^{-1}$, very similar to previous observations of near $-70 \mathrm{~W} \mathrm{~m}^{-2} \tau^{-1}$ (Yu et al., 2004; Zhang et al., 2005a). The regression based method produces a similar value, $-68.2 \mathrm{~W} \mathrm{~m}^{-2} \tau^{-1}$, in close agreement with the $-70 \mathrm{~W} \mathrm{~m}^{-2} \tau^{-1}$ value reported by Christopher and Zhang (2002) and the $-72 \mathrm{~W} \mathrm{~m}^{-2} \tau^{-1}$ value by $\mathrm{Yu}$ et al. (2004) (with diurnal adjustment removed). The AOTSWRE relationship, shown in Fig. 3, is approximately linear with a linear correlation coefficient of -0.93 . This indicates the model coefficient, which can also be interpreted as $E_{\tau}$, is a robust statistic and not very susceptible to outliers.

\subsection{Gridded data}

The primary reason of the overestimation of AOT and SWRE by the simple average is due to the spatial distribution of the data. Figure 4 shows gridded $\left(2 \times 2^{\circ}\right)$ instantaneous SWRE for the entire globe. It is evident from this figure that high values of are concentrated in a few geographic regions, most notably west of Africa between $10-20^{\circ} \mathrm{N}$, the East Coast of China, and the Bay of Bengal. These regions are also associated with the greatest variation in SWRE, which is evident in Fig. 5, which shows the standard deviation of SWRE within each grid-cell. Note that the regions of highest variability 


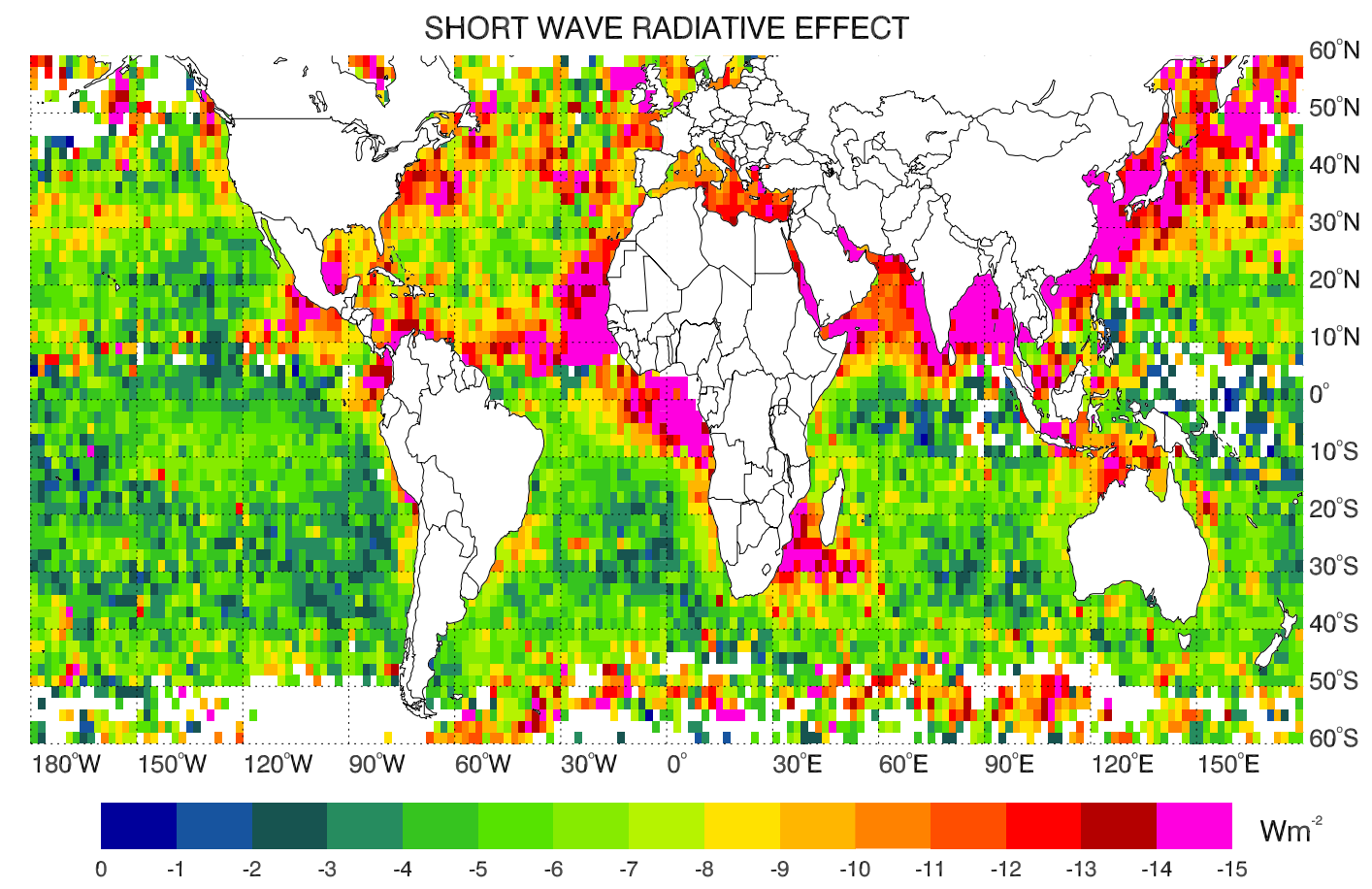

Fig. 4. Global SWRE gridded into $2 \times 2$ degree grid-cells.

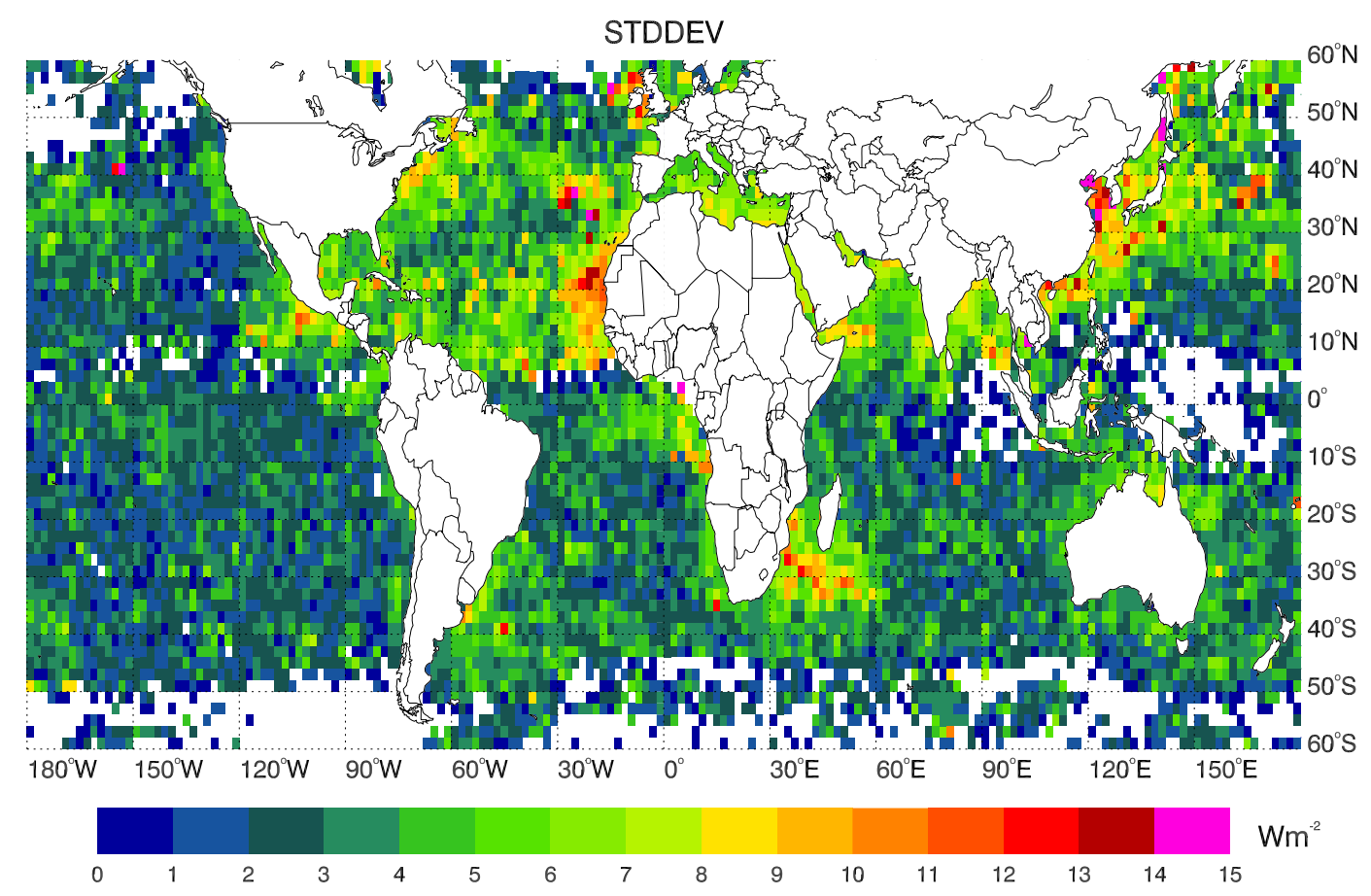

Fig. 5. Standard deviation of SWRE within each $2 \times 2$ degree grid-cell.

correspond with the regions of greatest SWRE. Grid-cells within these regions also contain the greatest number of raw pixels (Fig. 6). At first glance, it appears odd that valid data should be concentrated where only high aerosol con- centrations exist. However, this is a result of the use of only cloud-free data in this analysis. Figure 7 shows globally averaged MODIS cloud fraction for all data (cloud fraction threshold not applied). Note that the regions associated with 


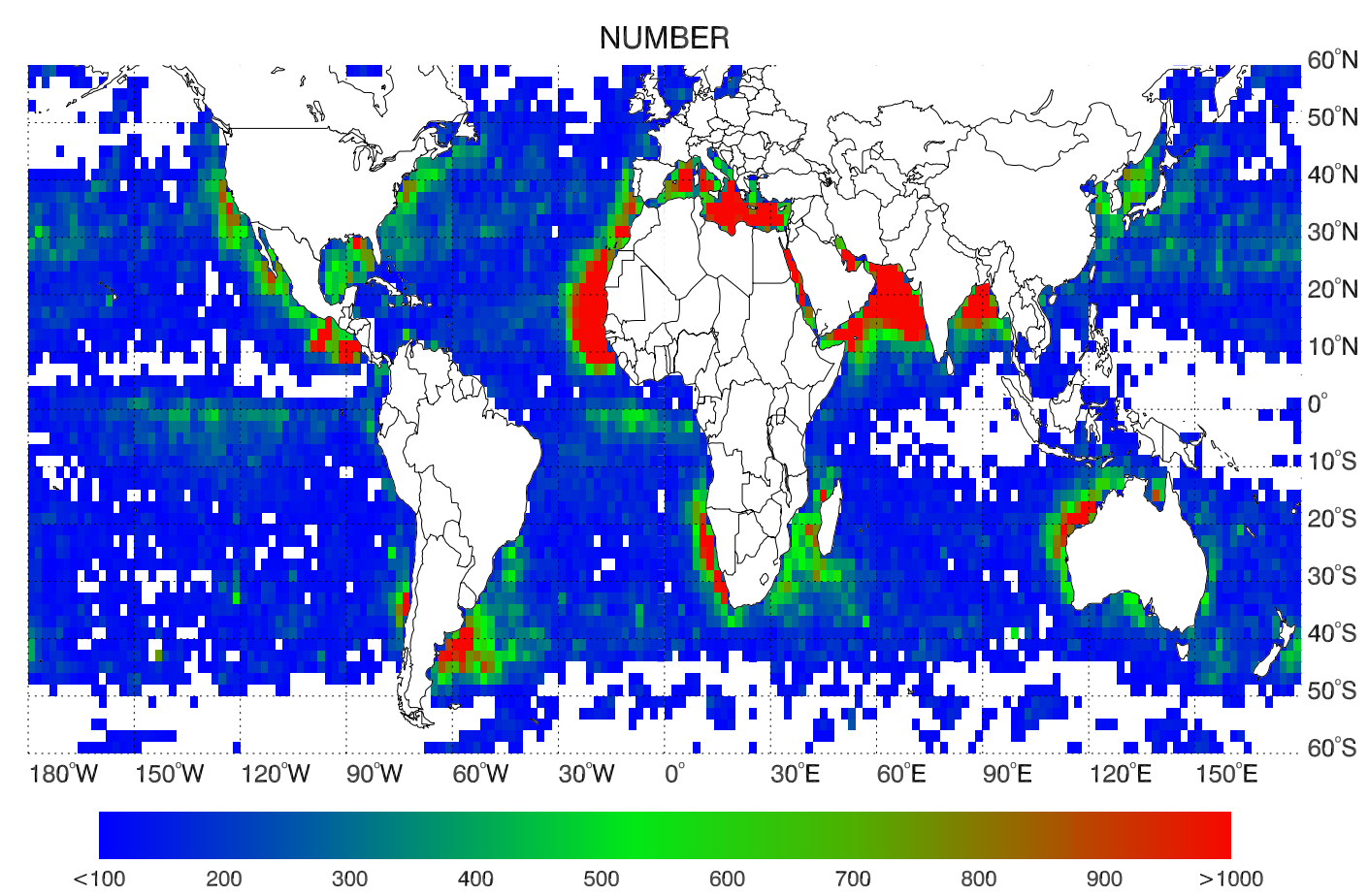

Fig. 6. Number of raw pixels present within each grid-cell.

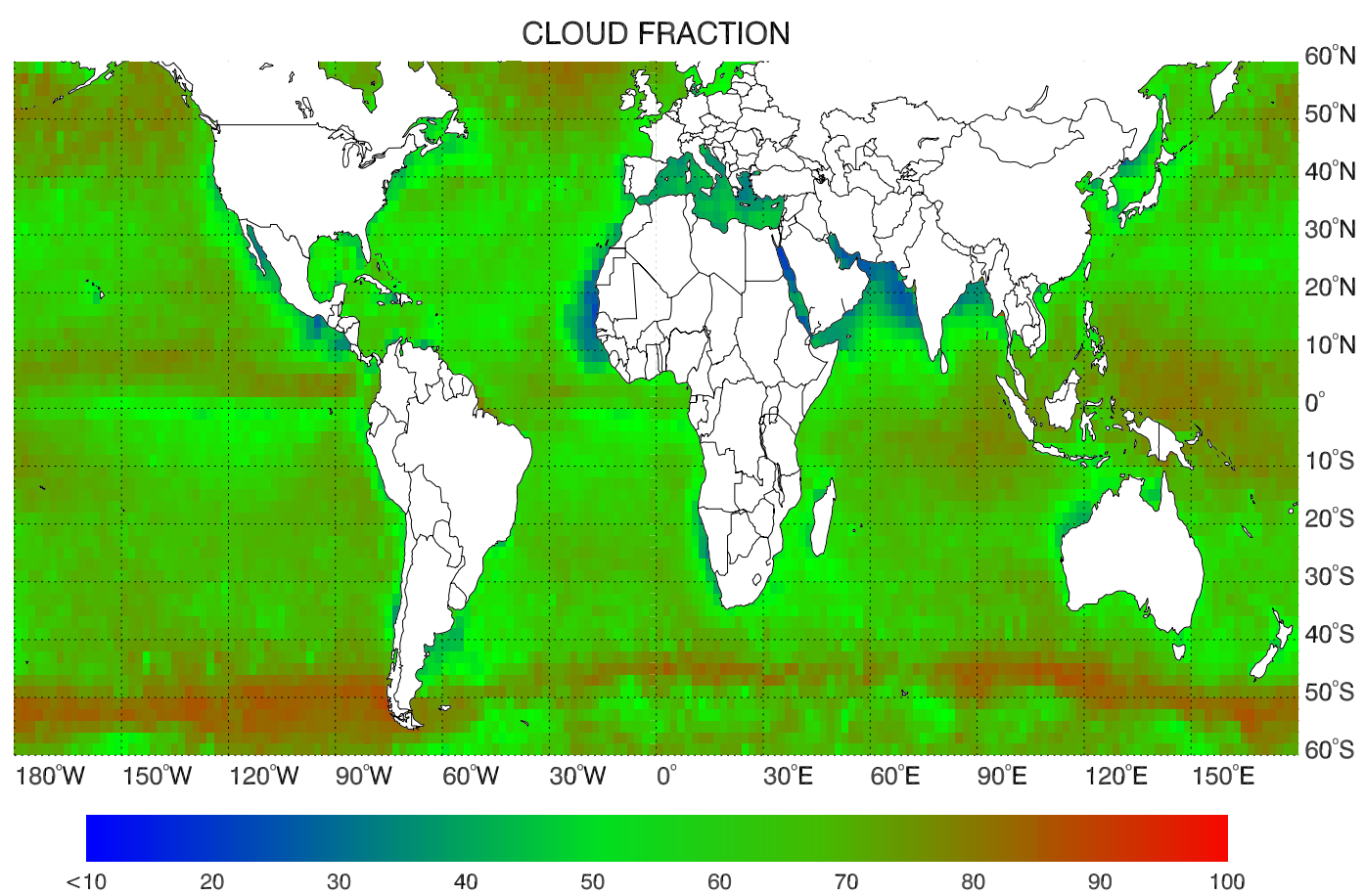

Fig. 7. Global MODIS cloud fraction for each grid-cell derived from clear and cloudy sky data.

high SWRE also happen to have low mean cloud fraction. As a result, data within these regions is more likely to pass the cloud-free threshold and included in the SWRE analysis. Since a greater number of pixels are available in high AOT and SWRE regions, globally averaged AOT and SWRE are overestimated using this averaging technique.

The use of clear-sky only data for the calculation of SWRE has several implications. We use cloud-free only data since 

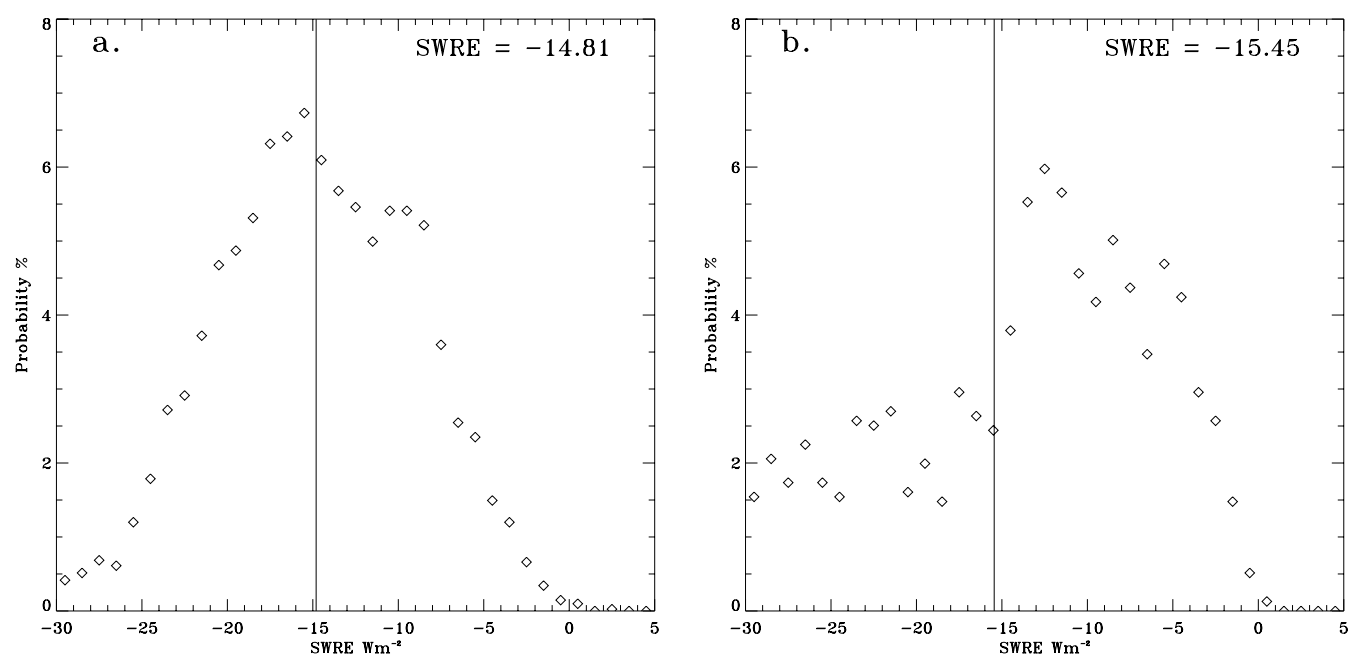

Fig. 8. Probability density function of raw data points present in selected grid-cells at $19^{\circ} \mathrm{N}, 69^{\circ} \mathrm{E}(\mathbf{a})$, and $19^{\circ} \mathrm{N}, 19^{\circ} \mathrm{W}(\mathbf{b})$.

the effect of clouds on atmospheric radiation are considered outside the scope of direct aerosol forcing research. However, the resolution of the CERES SSF product $(20 \times 20 \mathrm{~km})$ requires that a $400 \mathrm{~km}^{2}$ region must be almost completely clear to satisfy the cloud free criteria defined in Sect. 2. As a result, large regions which are predominately cloud-free contain a greater number of data points than to predominately cloudy regions. Comparing Figs. 4 and 7 confirms this with the greatest number of data points per grid cell being defined where cloud fraction is relatively low. Often (but not always), predominantly cloudy regions also have substantial concentrations of aerosols. Using the method presented here, these aerosols and their effect on incoming solar radiation are ignored, leading to an underestimation of SWRE. To account for this issue, Zhang et al. (2005b) created a "bias adjustment" technique using the AOT difference between high resolution MODIS AOT and CERES-SSF resolution AOT to adjust the global SWRE number. Since we focus on statistical variability alone, we refer discussion of the bias adjustment to Zhang et al. (2005b).

Upon gridding the raw data, sample size falls from nearly one million data points to approximately 7400 grid-cells (Table 1). The effect of changing grid-cell size to either 1 or 4 degrees was studied, and did not significantly change the results compared to 2 degree gridded resolution data. The number of raw pixels contained within each $2 \times 2$ degree grid cell ranges from 1 to 4085 . The grid-cell containing the most pixels (4085) is centered at $19^{\circ} \mathrm{N}$ and $69^{\circ} \mathrm{E}$ within the Arabian Sea. The SWRE for this grid-cell is $-14.8 \pm 5.8 \mathrm{~W} \mathrm{~m}^{-2}$, but with a skewness statistic of only -0.1 . For this grid-cell in particular, the raw pixels are approximately normally distributed indicating that the grid-cell average is representative in this case (Fig. 8a). Future research may be able to utilize regions where AOT and SWRE are normally distributed to compare results of differing research techniques, since the statistical variability is reduced compared to the global data set. However, there are many grid-cells that do not contain a normal distribution of data such as the grid-cell centered and $19^{\circ} \mathrm{N}, 19^{\circ} \mathrm{W}$, and are generally biased in a manner similar to the global data set (Fig. 8b). The SWRE distribution in this grid-cell is likely a reflection of different aerosol regimes present over this region, which can change from dust to anthropogenic depending on time of year (e.g. Ramana and Ramanathan, 2006).

No matter what the underlying cause, the gridded data set also suffers from a lack of a normal distribution. The globally averaged gridded statistics make this point evident. Gridded average AOT and SWRE are substantially lower than there non-gridded counterparts $(0.12,-8.0$ vs. 0.15 , $-10.6 \mathrm{~W} \mathrm{~m}^{-2}$ ) (Table 1). However, the skewness statistic for both gridded AOT and SWRE are higher, indicating that the gridded average is less representative than before.

The globally averaged gridded AOT and SWRE are substantially lower than their non-gridded counterparts since gridding removes the spatial bias towards data in high aerosol concentration regions. Thus, the gridded data set has a fewer number of high AOT pixels incorporated into the average. The more uniform spatial distribution creates a globally averaged instantaneous SWRE that is somewhat closer to the previously reported values of -6.4 and $-7.6 \mathrm{~W} \mathrm{~m}^{-2}$ (Zhang et al., 2005b; Loeb Manalo-Smith, 2005); however, the lack of a normal distribution leaves a large uncertainty as to the true globally averaged value. As with the raw data, the gridded data was randomly sampled to determine its resiliency to outliers. The results indicate that the gridded data set, like the raw data set before, is very resilient to outliers. AOT and SWRE also vary less $1 \%$ from their gridded mean values (Fig. 9). 

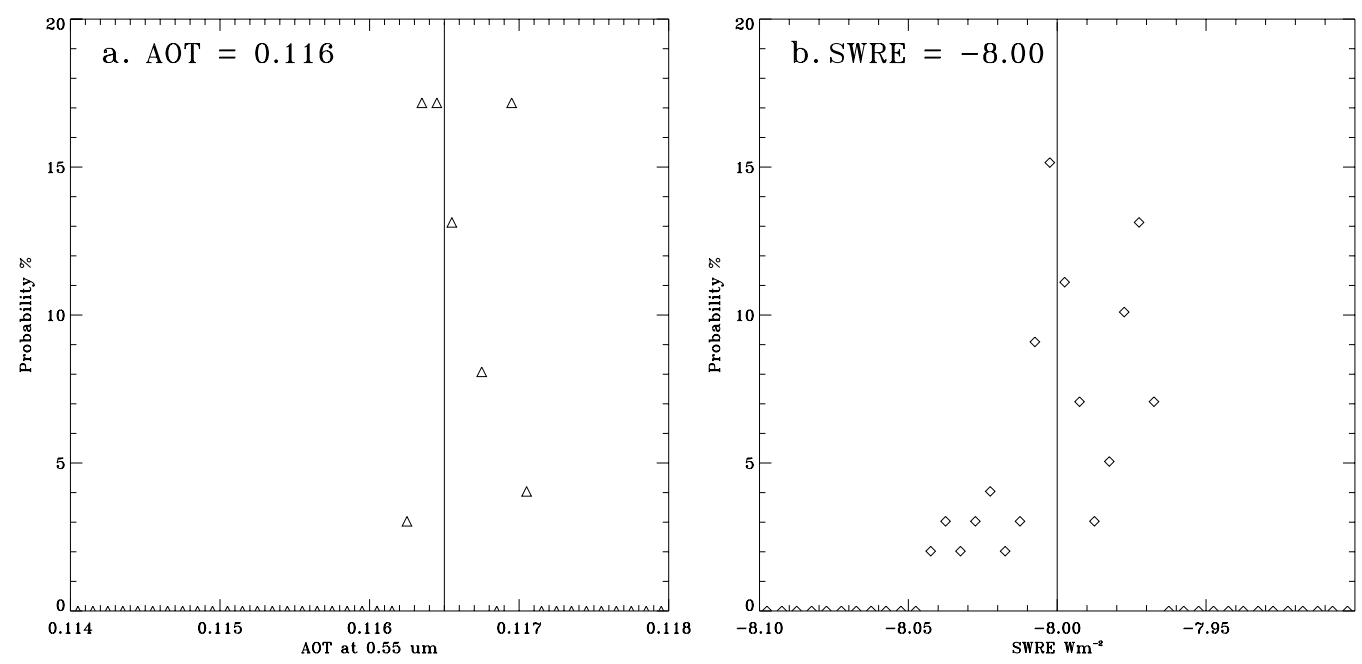

Fig. 9. Probability density function of randomly sampled gridded AOT (a) and SWRE (b) data.
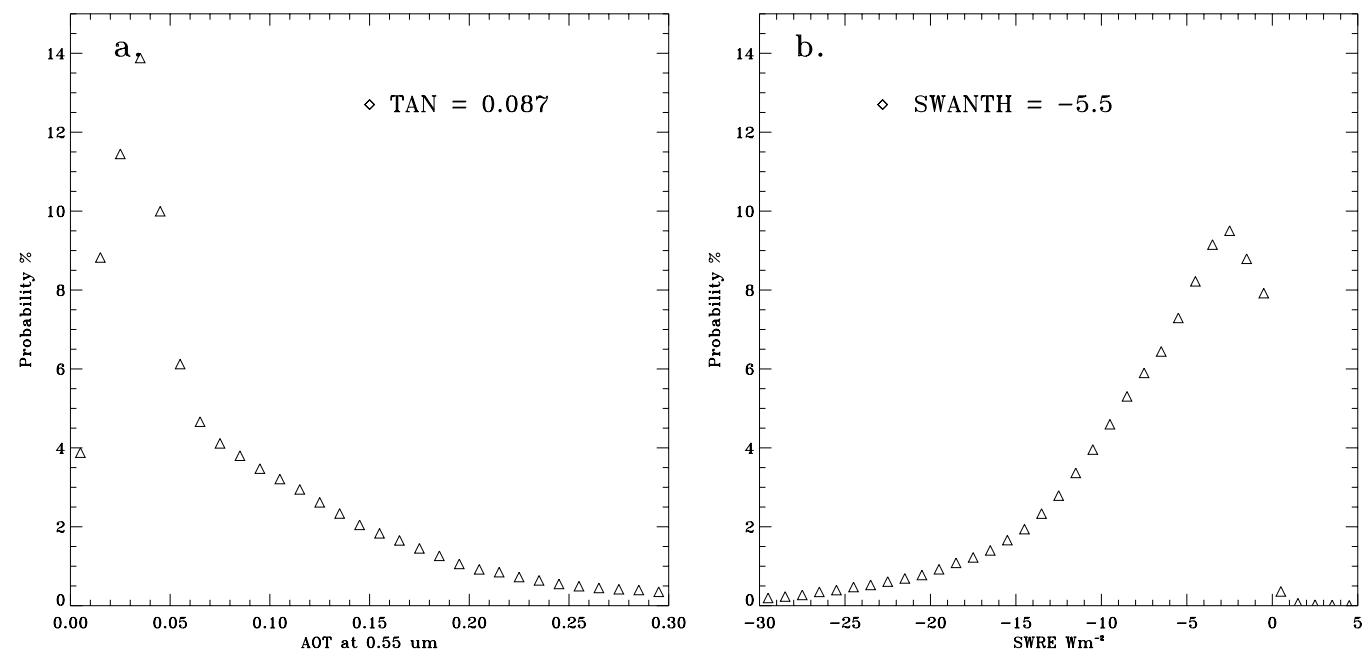

Fig. 10. Anthropogenic component AOT (a) and SWRE (b) probability density functions.

Calculation of $E_{\tau}$ using the gridded data set produces some interesting results. The traditional definition results in an $E_{\tau}$ value of $-68.4 \mathrm{~W} \mathrm{~m}^{-2} \tau^{-1}$, with the regression method producing a somewhat smaller value, $-60.8 \mathrm{~W} \mathrm{~m}^{-2} \tau^{-1}$. Using the traditional method, no significant difference in SWRE is apparent between raw and gridded data. However, the use of the regression method on gridded data underestimates $E_{\tau}$ by approximately $10 \%$ compared to other methods. The additional step of gridding results in an underestimation of SWRE for some grid-cells, which leads to an artificial change in the AOT-SWRE relationship. As a result, $E_{\tau}$ should not be calculated from gridded or otherwise manipulated data, but from the original raw data.

\subsection{Anthropogenic aerosols}

The methods described by Christopher and Jones (2007) and Sect. 2 were used to derive anthropogenic AOT and SWRE over a global domain for both gridded and nongridded data (Table 2). As with total aerosol AOT and SWRE, anthropogenic AOT and SWRE also lack a normal distribution with anthropogenic AOT probability maximized at very low values (Fig. 10). Non-gridded average anthropogenic AOT and instantaneous SWRE are quite high $(0.08$, $-7.6 \mathrm{~W} \mathrm{~m}^{-2}$ ) compared to previously reported values of 0.033 and $-2.8 \mathrm{~W} \mathrm{~m}^{-2}$ (these values have been converted to instantaneous) by both Kaufman et al. (2005b) and Christopher et al. (2006). The high values reported here are the result of two factors. First, as with total AOT, more anthropogenic 
aerosol data points exist in regions of heavy aerosol concentrations due to the greater probability of cloud-free observations being present (Figs. 6, 7). The second is that the averages presented above do not take into account regions where no anthropogenic aerosols exist. Christopher et al. (2006) set anthropogenic AOT and SWRE in these regions equal to 0 prior to computing their global averages. Applying this assumption to the non-gridded data results in globally averaged values of 0.07 and $-5.0 \mathrm{~W} \mathrm{~m}^{-2}$ (Table 2).

Statistics for gridded anthropogenic AOT and SWRE are also reported (Table 2). Two sets of gridded data were generated, one including only data where are particular aerosol type exists and the other incorporating the null assumption for data points where anthropogenic aerosols did not exist. As with the non-gridded data, the use of this assumption reduces globally averaged AOT and SWRE, to 0.04 and $-3.2 \mathrm{~W} \mathrm{~m}^{-2}$ when including null data in the gridding process (Table 2). These values are much closer to those reported by Kaufman et al. (2005b) and Christopher et al. (2006).

Additional variability in anthropogenic AOT and SWRE results from the method used to separate the anthropogenic component. Recall that anthropogenic AOT was defined as the residual of total AOT, dust, and maritime components. Thus, any AOT remaining after dust and maritime components have been calculated is assigned as anthropogenic. This assumption can represent an artificial increase in the importance of anthropogenic aerosols relative to the other components. Kaufman et al. (2005b) do solve Eqs. (1-5) for anthropogenic AOT instead of dust AOT. However, the resulting anthropogenic equation is not $100 \%$ mathematically consistent with the dust equation (Eq. 4). Here, dust AOT is calculated using the residual method, artificially increasing its importance relative to anthropogenic AOT. The only solution to this would be to develop mathematically consistent equations for the calculation of dust and anthropogenic AOT, which is left for future research.

\section{Conclusions}

The question remains as to what is the actual TOA instantaneous shortwave radiative effect from aerosols over a global domain. This work has shown that significantly different values can be returned depending on the technique used to analyze the data. No one method for calculating globally averaged AOT and SWRE generates values without substantial statistical uncertainty. The "raw data" average suffers from a non-uniform spatial distribution, weighted towards regions with high aerosol concentrations. As a result, statistics derived from these data overestimate global AOT and SWRE, despite the probability distribution favoring lower values of both. The gridded data is close to spatially uniform (over ocean regions), and the resulting average does not show the large high bias produced by the non-gridded data. However, the distribution of raw data within each grid-
Table 2. Non-gridded and gridded, globally averaged anthropogenic AOT and SWRE for datasets that include and do not include null data where anthropogenic AOT do not exist. The difference in sample size from AOT to SWRE is due to the constraint that anthropogenic AOT can only occur for data where $0.3<f<0.9$.

\begin{tabular}{crr}
\hline & AOT & SWRE \\
\hline$N$ & 812257 & 583983 \\
Non-null & 0.084 & -7.563 \\
Null & 0.068 & -5.033 \\
$N$ & 7252 & 6256 \\
$N$ & 0.057 & -4.65 \\
Grid & 0.043 & -3.196 \\
Null-Grid & \\
\hline
\end{tabular}

cell leads to significant uncertainty as to some grid-cell values. Grid-cells with non-uniform distributions are generally biased toward low AOT and SWRE values, with the resulting grid-cell average likely being an underestimate of the actual value. Thus, we are left with a range of values for global AOT and instantaneous SWRE $(0.12<$ AOT $<0.15$; $-8.0<\mathrm{SWRE}<-10.6 \mathrm{~W} \mathrm{~m}^{-2}$ ), with true values likely lying somewhere in between, which represents an uncertainty of over $25 \%$. Due to the much greater probability of aerosol data existing in aerosol-heavy regions, the actual value for AOT and SWRE lies closer to the gridded value than the non-gridded value. Just how close remains uncertain, but the gridded dataset produces more realistic results. Fortunately, the random sample analysis for both gridded and non-gridded datasets showed that globally averaged values are not susceptible to outliers present in the data.

Attempting to determine maritime, dust, and, anthropogenic component statistics is even more difficult depending on the treatment of regions where one or more aerosol types do not exist. Including regions of null-data in the computation of a globally averaged mean reduces anthropogenic AOT and SWRE. The magnitude of this decrease is also uncertain as it relies on the method used to separate AOT components (see Sect. 4c). When reporting globally averaged component values, it is vital to state whether or not null data are being included and show distribution of component data. Otherwise, it is impossible to determine the significance of any reported globally averaged AOT or SWRE statistics.

The primary conclusion of this work is that a globally average AOT or SWRE value is not meaningful unless additional statistics are supplied. It is important to know the underlying distribution of the sample data as well as its spatial and temporal variability. When dealing with observational datasets such as the one used here, data are not likely to be present on a uniform spatial or temporal domain. Eliminating this issue requires that the raw data be placed onto a uniform as described in Sect. 3c. The resulting globally averaged AOT and SWRE are much closer to previously reported values, but significant statistical uncertainties remain. 
No single averaging technique or statistics provides an adequate description of global AOT and SWRE.

Only a combination of statistical and distribution information can provide an estimate as to the magnitude and uncertainty of globally averaged values. In addition to globally averaged values, future works should also report data distribution statistics, such as skewness and probability density functions. When non-normal distributions are encountered, its needs to be reported what effect the actual distribution will have on a globally averaged value. Also important to note are all forms of data manipulation, such as gridding, diurnal/sample bias adjustments performed on the data. Manipulating data in various ways often improves its statistical quality, but can also change its underlying meaning. Without this rigorous documentation, results from various works, even when done over the same spatial and temporal domain may not be comparable.

Acknowledgements. This research was supported by NASA's Radiation Sciences, NASA Interdisciplinary Sciences, NASA EOS and NASA ACMAP programs. The CERES data was obtained from the NASA Langley Research Center Atmospheric Sciences Data Center, the MODIS data were obtained through the Goddard Space Flight Center Data Center.

Edited by: A. Nenes

\section{References}

Anderson, T. L., Charlson, R. J., Bellouin, N., Boucher, O., Chin, M., Christopher, S. A., Haywood, J., Kaufman, Y. J., Kinne, S., Ogren, J. A., Remer, L. A., Takemura, T., Tanré, D., Torres, O., Trepte, C. R., Wielicki, B. A., Winker, D. M., and Yu, H.: An "A-Train" Strategy for Quantifying Direct Climate Forcing by Anthropogenic Aerosols, Bull. Amer. Meteorol. Soc., 86, 17951809, 2005.

Bellouin, N., Boucher, O., Haywood, J., and Reddy, M. S.: Global estimate of aerosol direct radiative forcing from satellite measurements, Nature, 438, 1138-1141, doi:10.1038/nature04348, 2005.

Christopher, S. A. and Zhang, J.: Shortwave aerosol radiative forcing from MODIS and CERES observations over the oceans, Geophys. Res. Lett., 29, 1859, doi:10.1029/2002GL014803, 2002.

Christopher, S. A., Zhang, J., Kaufman, Y. J., and Remer, L. A.: Satellite-based assessment of top of atmosphere anthropogenic radiative forcing over cloud-free oceans, Geophys. Res. Lett., 33, L15816, doi:10.1029/2005GL025535, 2006.
Christopher, S. A. and Jones, T. A.: Satellite-based Assessment of Cloud-free Net Radiative Effect of Dust Aerosols over the Atlantic Ocean, Geophys. Res. Lett., 34, L02810, doi:10.1029/2006GL027783, 2007.

Kaufman, Y. J., Koren, I., Remer, L. A., Tanré, D., Ginoux, P., and Fan, S.: Dust transport and deposition observed from the Terra-Moderate Resolution Imaging Spectroradiometer (MODIS) spacecraft over the Atlantic Ocean, J. Geophys. Res., 110, D10S12, doi:10.1029/2003JD004436, 2005a.

Kaufman, Y. J., Boucher, O., Tanré, D., Chin, M., Remer, L. A., and Takemura, T.: Aerosol anthropogenic component estimated from satellite data, Geophys. Res. Lett., 32, L17804, doi:10.1029/2005GL023125, 2005b.

Li, F., Vogelmann, A. A., and Ramanathan, V.: Saharan Dust Radiative Forcing Measured from Space, J. Climate, 17, 2558-2571, 2004.

Loeb, N. G. and Manalo-Smith, N.: Top-of-atmosphere direct radiative effect of aerosols over global oceans from merged CERES and MODIS observations, J. Climate, 18, 3506-3526, 2005.

Ramana, M. V. and Ramanathan, V.: Abrupt transition from natural to anthropogenic aerosol radiative forcing: Observations at the ABC-Maldives Climate Observatory, J. Geophys. Res., 111, D20207, doi:10.1029/2006JD007063, 2006.

Wilks, D. S.: Statistical Methods in the Atmospheric Sciences, Academic Press, 627 pp., 2006.

Yu, H., Dickinson, R. E., Chin, M., Kaufman, Y. J., Zhou, M., Zhou, L., Tian, Y., Dubovik, O., and Holben, B. N.: The direct radiative effect of aerosols as determined from a combination of MODIS and GOCART simulations, J. Geophys. Res., 109, D03206, doi:10.1029/2003JD003914, 2004.

Yu, H., Kaufman, Y. J., Chin, M., Feingold, G., Remer, L. A., Anderson, T. L., Balkanski, Y., Bellouin, N., Boucher, O., Christopher, S., DeCola, P., Kahn, R., Loeb, N., Reddy, M. S., Schulz, M., Takemura, T., and Zhou, M.: A review of measurementbased assessment of aerosol direct radiative effect and forcing, Atmos. Chem. Phys. 6, 613-666, 2006.

Zhang J., Christopher, S. A., Remer, L. A., and Kaufman, Y. J.: Shortwave aerosol radiative forcing over cloud-free oceans from Terra: 2: Seasonal and global distributions, J. Geophys. Res., 110, D10S24, doi:10.1029/2004JD005009, 2005a.

Zhang, J., Christopher, S. A., Remer, L. A., and Kaufman, Y. J.: Shortwave aerosol radiative forcing over cloud-free oceans from Terra: Angular models for aerosols, J. Geophys. Res., 110, D10S23, doi:10.1029/2004JD005008, 2005b.

Zhang, J. and Reid, J. S.: MODIS aerosol product analysis for data assimilation: Assessment of over-ocean level 2 aerosol optical thickness retrievals, J. Geophys. Res., 111, D22207, doi:10.1029/2005JD006898, 2006. 